\title{
Conectividad con la naturaleza y conducta sustentable: Una vía hacia las conductas pro-sociales y pro-ambientales
}

\section{Connectedness to nature and sustainable behavior: A path towards pro-social and pro-environmental behaviors}

Fernanda Inéz García Vázquez

Maria Fernanda Durón Ramos

Instituto Tecnológico de Sonora
Víctor Corral Verdugo

Universidad de Sonora

\section{Resumen}

Al tomar como base la idea de que la Conectividad con la Naturaleza $(\mathrm{CN})$ es esencial para afrontar los problemas ambientales, el objetivo del presente estudio fue demostrar la hipótesis de que las personas con una mayor conexión con la naturaleza realizan más Conductas Sustentables (CS). Estudios previos han reportado que este sentido de conexión podría aumentar la práctica de comportamientos proambientales; sin embargo, hasta el momento, no existen investigaciones que consideren la relación entre los cuatro componentes de la CS (Altruismo, equidad, austeridad y conducta pro-ambiental) y la CN. En este estudio participaron 207 residentes de la ciudad de Hermosillo, México, quienes contestaron un instrumento que mide las cuatro instancias de CS y una escala de CN. Para analizar las relaciones entre estas variables se especificó y probó un modelo de ecuaciones estructurales que reveló una relación significativa entre la conectividad con la naturaleza y las CS, lo cual sugiere que las personas con un fuerte sentido de conectividad hacia lo natural llevan a cabo un mayor número de comportamientos pro-ambientales, pero también pro-sociales. Estudios subsecuentes, donde se estudie de manera más profunda este principio y sus implicaciones, son necesarios para profundizar en las repercusiones de la CS.

Palabras clave: conectividad con la naturaleza, conducta sustentable, conducta pro-ecológica, altruismo, austeridad, equidad.

Nota del autor

Fernanda Inéz García Vázquez, Departamento de Educación, Instituto Tecnológico de Sonora; Maria Fernanda Durón Ramos, Departamento de Psicología, Instituto Tecnológico de Sonora unidad Guaymas; Víctor Corral Verdugo, Posgrado Integral en Ciencias Sociales, Universidad de Sonora.

La correspondencia en relación con este artículo debe dirigirse a Fernanda Inéz García Vázquez, Departamento de Educación, Instituto Tecnológico de Sonora, Edificio CEVE, Campus Centro, 5 de febrero 818 sur, C.P. 85000. Ciudad Obregón, Sonora. México.

Dirección electrónica: fernanda.garcia@itson.edu.mx 


\begin{abstract}
Grounded on the idea that connectedness to nature is essential to facing environmental problems, the aim of this study was to demonstrate the hypothesis that people who perceive a greater sense of connectedness with nature will practice more sustainable behaviors (SB) Previous studies had reported that this sense of connectedness might enhance the practice of pro-environmental behaviors. Yet, so far no study considering the relationship between an aggregate of the four instances of SB (pro-ecological, altruistic, frugal and equitable behaviors) and connectedness to nature, had been conducted. Two hundred seventy-seven individuals at the city of Hermosillo, Mexico responded to an instrument assessing the four instances of SB, as well as a connectedness to nature scale. A structural equation model revealed a significant relation between connectedness to nature and SB, which suggests that people with higher connection to nature will practice more pro-environmental but also pro-social behaviors. Further studies, in which this connectedness and its implications are studied more deeply, are required.
\end{abstract}

Keywords: connectedness to nature, sustainable behavior, pro-ecological behavior, altruism, frugality, equity.

La humanidad se enfrenta a una de las crisis ambientales y sociales más agudas en su historia, con un deterioro de la biosfera, que pudiera ser irreversible, y disparidades sociales que son cada vez más notorias. Indudablemente, muchas de estas problemáticas tienen una estrecha relación con el estilo de vida y la manera en la que entramos en contacto con la naturaleza. Para algunos investigadores como Clayton (1998), Frantz, Mayer, Norton y Rock (2005) y Schultz, Shriver, Tabanico y Khazian (2004), la respuesta a esta crisis se encuentra en el sentido de $\mathrm{CN}$. La conexión con la naturaleza sería necesaria al buscar posibles soluciones a las cuestiones ambientales; además, creciente literatura que documenta la importancia de esta conexión hace hincapié en la necesidad de permanecer integrado(a)s con el entorno y no separado(a)s de él (Cronon, 1995; Levi
\& Kocher, 1999; Dutcher, Finley, Luloff, \& Johnson 2007).

Dutcher, Finley, Lullof y Johnson (2007) aseguran que los seres humanos que sienten una igualdad fundamental entre ellos y el mundo natural (así como con otras personas) experimentan más empatía y compasión hacia la naturaleza. Por lo anterior, la falta de $\mathrm{CN}$ equivale a alienación de la naturaleza, la cual se manifiesta en una perspectiva dominante. Levi y Kocher (1999), por su parte, sugieren que la desconexión de la naturaleza es peligrosa debido a su impacto en los seres humanos $y$ el medio ambiente, ya que somos menos conscientes de lo que estamos haciendo contra el ambiente. Cronon (1995), en tanto, sostiene que la gente debería dejar de poner límites entre ella y la naturaleza. Además, señala que con el fin de proteger con éxito todo el ambiente, no 
sólo a pequeñas partes de él, hay que eliminar las barreras percibidas. Melson (2001), por su parte, argumenta que con la desaparición de especies y la diversidad de hábitats, se niega a las niñas y niños el sentido de conexión, con lo cual se formará una nueva generación que carece de una convicción profunda de lo que es un ambiente compartido y, debido a esto, es sólo cuestión de tiempo para acelerar aún más la degradación ecológica.

Se sabe, por trabajos anteriores, que el nivel de conexión que siente un individuo hacia un entorno afectará el nivel del interés y la gestión de las decisiones hacia el ambiente (Schroeder, 2002; Schultz, 2000). Un número creciente de investigaciones demuestra que la $\mathrm{CN}$ se asocia fuertemente con la preocupación ambiental y el comportamiento responsable con el medio ambiente (Schultz, 2001; Hinds \& Sparks, 2008; Müller, Kals, \& Pansa, 2009; Brugger, Kaiser, \& Roczen, 2011).

Hay que considerar, además, que en estos días la conducta ambiental relevante ya no se considera sólo pro-ambiental o protectora del ambiente físico sino sustentable, término que incluye la protección del componente social del ambiente, además del elemento físico. Esta conducta es indicada por una serie de dimensiones psicológicas, entre las cuales se encuentran la equidad, el altruismo, la proecología y la austeridad (Tapia, Corral, Fraijo \& Durón, 2013).

Como se ha argumentado anteriormente, la conectividad es un factor muy importante al considerar la conservación de los recursos naturales y socio-culturales; por lo tanto, el objetivo de la presente investigación es demostrar que las personas con un mayor sentido de $\mathrm{CN}$, realizarán más conductas sustentables.

\section{La experiencia de conectividad}

Schultz et al. (2004) afirman que las personas que sienten las conexiones más fuertes con la naturaleza expresan mayor preocupación por la biosfera y reportan participar en comportamientos que ayudan a proteger el medio ambiente. Sentirse conectado con la naturaleza contribuye a generar un sentimiento de responsabilidad y de cuidado de otras criaturas y del entorno natural (Taylor, 1989).

Clayton (1998) sostiene que sólo la experiencia de conectividad salvará la tierra y a los seres humanos con ella. Menciona, además, que cualquier intento no será suficiente si no se basa en la transformación de la experiencia en la que el pensamiento humano conoce la conectividad como tal. Posteriormente, dicha autora desarrolla una escala de identidad ambiental para evaluar cómo el medio ambiente natural juega un papel en la autodefinición de una persona. Ella propone que la identidad ambiental forma parte de los elementos con los cuales las personas configuran su autoconcepto, un sentido de conexión con el medio ambiente natural, basado en la historia, los lazos emocionales, y la similitud, que afecta las formas en que percibimos y actuamos hacia el mundo: la creencia de que el medio ambiente es 
importante para nosotros y una parte importante de lo que somos (Clayton, 2003).

Del mismo modo, Fisher (2002) menciona que una fuerte conexión afectiva y/o espiritual con la naturaleza en sus infinitas expresiones puede fomentar valores positivos ambientales, actitudes y comportamiento pro-ambientales. En este mismo sentido, Maller, Townsend, St. Leger, Henderson-Wilson, Pryor, Prosser y Moore (2008) afirman que las conexiones espirituales con la naturaleza se presentan a través de enfocarse en la pasión que las personas tienen por restaurar los ambientes naturales y de conectarse con sus comunidades, por medio del cuidado de la Tierra.

Para Schultz (2000), el nivel personal de preocupación por el medio ambiente se encuentra directamente relacionado con el sentido de conexión que el individuo siente con la naturaleza. De igual manera, Nisbet, Zelenski y Murphy (2009) argumentan que la desconexión con el mundo natural puede estar contribuyendo a la destrucción del planeta y proponen un nuevo constructo llamado "relación con la naturaleza", que incluye aspectos afectivos, cognitivos y experienciales.

De acuerdo a Corral (2012), para la conservación del ambiente socio-físico se requiere de un enfoque global, un sentido de conexión con la naturaleza, el empleo de una perspectiva ampliada para la acción y el pensamiento, la aplicación de recursos instrumentales (físicos), intelectuales y sociales, así como una perspectiva temporal a largo plazo. Del mismo modo, muchas tradiciones filosóficas, religiosas y culturales han promovido la idea de que las personas están intrínsecamente conectadas con otros seres humanos y con el mundo natural (Leary, Tipsord, \& Tate, 2008). Por ejemplo, la noción de interconexión con el entorno se enfatiza en las religiones orientales como el budismo; esta noción afirma que las fronteras entre uno mismo y los otros, como entre uno mismo y el medio ambiente, son borrosas o incluso inexistentes (Nhat Hahn, 1999).

Ciertas culturas fomentan, asimismo, una mayor afiliación con la naturaleza; por ejemplo, existe un arraigo profundo en las culturas de Noruega y Suecia al concepto de friluftsliv, una palabra cuya traducción significa "vida al aire libre", un estilo de vida filosófico, basado en la experiencia de libertad en la naturaleza y una conectividad espiritual con el paisaje (Gelter, 2000).

Existe teoría e investigación a través de los años que da cuenta de esta conexión. De hecho, hay cierta evidencia de que los seres humanos han evolucionado para sentir una conexión especial con el mundo natural (Davis, Green, \& Reed, 2009). Según Wilson (1983), el cerebro evolucionó en un mundo biocéntrico y no en un mundo regulado por las máquinas, en consecuencia, sería muy difícil aceptar que todas las reglas aprendidas, relacionadas con ese mundo, se hayan borrado en algunos cientos de miles de años, aun en una apretada minoría de personas que han existido por una o dos generaciones en ambientes totalmente 
urbanos. Al respecto, Wilson había hablado ya (1983), al definir la biofilia como una tendencia evolucionada a experimentar el ambiente natural y una atracción hacia la vida. Por su parte Schultz y colaboradores (2004) afirman que la conexión que un individuo siente con la naturaleza es implícita o inconsciente.

Siguiendo con esta idea, la hipótesis de la biofilia afirma que los seres humanos poseen una atracción hacia la naturaleza basada en la biología y que su bienestar depende, en gran medida, de las relaciones con el mundo natural que los rodea. Como resultado de ello, los seres humanos necesitan afiliarse a la naturaleza (Kellert, 1997).

\section{EI concepto de Conectividad con la Naturaleza} (CN)

El concepto de $\mathrm{CN}$ ha sido definido $\mathrm{y}$ estudiado en diversas ocasiones y maneras. Estas definiciones tienen aspectos comunes y muestran grandes similitudes, sobre todo el sentimiento de unidad o cercanía con la naturaleza y su consiguiente protección, lo cual reflejaría la esencia del concepto. Para Olson y Zanna (1993), la conexión con la naturaleza presupone que los individuos tienen una "creencia primitiva" y respeto por la naturaleza que consiste en rasgos endógenos y disposicionales con una relativa estabilidad en el tiempo.

La noción de estar conectado con la naturaleza es psicológica, y el grado de conexión de un individuo tiene componentes cognitivos, afectivos y de comportamiento
(Schmuck \& Schultz, 2002). Uno de los estudios más representativos al respecto es el de Mayer y Frantz (2004). En dicho estudio, los autores definen, tal relación como la conexión emocional de un individuo con el mundo natural. Para Schultz (2002), esta conexión se refiere al grado en que un individuo incluye a la naturaleza dentro de la representación cognitiva de sí mismo.

La experiencia de conectividad implica la disolución de los límites y el sentido de una esencia compartida o común entre el yo, la naturaleza y otros (Dutcher, Finley, Luloff, \& Johnson, 2007). Para Hedlund, DeBoer y Boersema (2014), la conexión con la naturaleza es la expresión de un sentido personal de conexión con el medio natural y el cuidado del mismo. También parece implicar la preferencia por un determinado estilo de vida consciente y más natural, así como una actitud de querer contribuir para que eso sea posible. I-Chun, William y Chun-Yen (2014) plantean que este concepto describe un profundo aprecio y afiliación a entornos naturales. Del mismo modo, parecería que es poco probable que se produzca el cuidado ambiental en ausencia de la conexión con la naturaleza (Oberkircher, Shanafield, Ismailova, \& Saito, 2011).

De acuerdo con Dutcher, Finley, Lullof y Johnson (2007), la conectividad se refiere a la conducta de ayuda a uno mismo y a los demás, en el contexto de una disminución de la conciencia de la distinción entre los dos. Dicha CN puede ser conceptualizada, entonces, 
como la comprensión de la gente y la naturaleza como parte de la misma comunidad. Lo anterior significaría que la conectividad con la naturaleza debería impulsar el cuidado del ambiente natural; físico, pero también la protección del ambiente socio-cultural.

\section{Conducta sustentable (CS)}

El concepto de CS, al igual que las dimensiones del desarrollo sustentable, no sólo incluye acciones de cuidado del ambiente físico, sino, además, comportamientos y predisposiciones a cuidar a otras personas y a limitar el consumo de recursos (Corral, \& Pinheiro, 2004). La CS se define como el conjunto de acciones efectivas y deliberadas que tiene como finalidad el cuidado de los recursos naturales y socioculturales necesarios para garantizar el bienestar presente y futuro de la humanidad (Corral, \& Pinheiro, 2004). Las conductas sustentables, de acuerdo a Corral (2010), contienen las dimensiones de equidad, altruismo, pro-ecología y austeridad.

La equidad puede entenderse como la justicia que corresponde con los derechos o las leyes naturales, más específicamente con el hecho de liberarse de los sesgos o del favoritismo (Corral, 2010). Implica distribuir recursos de manera justa y tratar a los demás sin sesgos debidos a sus características demográficas o físicas. El altruismo hace referencia al funcionamiento por el cual las personas actúan a favor de sus semejantes de forma desinteresada, es decir, sin la expectativa de una acción recíproca de gratificación (García, Estévez, \& Letamendía,
2007). Para De Young (1991), la austeridad implica evitar de manera deliberada el consumo personal e innecesario de recursos. Se define como el uso prudente de los recursos y el interés por evitar el desperdicio. Puede llegar a constituirse en un estilo de vida "ligero". Las conductas pro-ecológicas, finalmente, son un conjunto de acciones deliberadas y efectivas que responde a requerimientos sociales e individuales y que resultan en la protección del medio ambiente natural (Corral, 2010).

\section{La CN y la CS}

Existen indicios de que la $\mathrm{CN}$ se relaciona con la práctica de conductas sustentables. Por ejemplo, Schultz (2001) desarrolló una escala de inclusión de la naturaleza en el self y encontró que la conectividad con la naturaleza se correlacionaba con la preocupación por la biosfera y el comportamiento ecológico autoreportado por los participantes. Este mismo autor llegó a la conclusión de que cualquier actividad que reduce la separación percibida de una persona entre ella misma y la naturaleza dará lugar a un aumento en la preocupación de ese individuo por la biosfera (Schultz, 2002).

Del mismo modo, Wells y Lekies (2006) encontraron que la participación en actividades en la naturaleza salvaje (caminatas, acampar) y actividades en la naturaleza interna (relativas a las flores y la jardinería) durante la niñez, se relacionan con las actitudes y comportamientos pro-ambientales en la edad adulta. Estos autores sugieren que la educación ambiental 
temprana parece tener un efecto sostenible en conexión con la naturaleza, asimismo, es probable que dé lugar a adultos ecológicamente responsables. Siguiendo con esta idea, Olivos, Aragonés y Navarro (2013) llevaron a cabo un estudio mediante un cuestionario que medía la conectividad, la preocupación ambiental (creencias ambientales) y la conducta proambiental. Estos autores encontraron una asociación positiva entre la conectividad y el comportamiento pro-ambiental.

Hinds y Sparks (2008) realizaron una investigación cuyas hipótesis afirmaban que la conexión afectiva y la identificación con el entorno natural contribuirían significativamente a la predicción de las intenciones de la gente. Estos autores encontraron que dicha relación afectiva era un predictor de las intenciones de comprometerse con el cuidado del medio ambiente natural.

Brugger, Kaiser y Roczen (2011) estudiaron, de igual forma, la $\mathrm{CN}$; los resultados que obtuvieron indican que las experiencias placenteras en la naturaleza promueven una conexión personal con el medio natural y ésta, a su vez, hace más posible la conservación del medio natural. Hedlund, DeBoer y Boersema (2014) llevaron a cabo un estudio sobre cómo las actitudes ambientes y los estilos de vida sustentables se relacionan con las visiones de mundo. Los resultados mostraron que el crecimiento interior y la espiritualidad contemporánea se relacionan con la conexión con la naturaleza y la voluntad de cambio (estilos de vida más sostenibles).

Estudios previos como los mencionados en párrafos precedentes, han reportado que la conectividad con el medio natural podría incrementar la práctica de comportamientos proambientales y haría más posible la conservación ambiental y la preservación de la vida en la tierra. Hace falta, sin embargo, comprobar si la relación entre la $\mathrm{CN}$ y el cuidado del ambiente natural se expande al cuidado del ambiente social. Por lo anterior, en el presente estudio se investigan las posibles asociaciones entre dicha conectividad y la conservación de los recursos socio-físicos del entorno, conservación concebida como conducta sustentable. En el presente trabajo se toma en consideración la relación entre los cuatro componentes de la conducta sustentable (altruismo, equidad, austeridad y conducta proambiental) y la $\mathrm{CN}$.

\section{Método}

\section{Participantes}

El presente estudio se realizó con un diseño cuantitativo de tipo transversal, con una población de 277 personas, seleccionadas al azar en zonas representativas de tres estratos sociales (alto, medio, bajo) de la ciudad de Hermosillo, Sonora. Estas fueron seleccionadas por medio de un muestreo no probabilístico; se eligieron sujetos típicos con la finalidad de que sean 
representativos de una población determinada (Hernández, Fernández, \& Baptista, 2010). De la muestra $41 \%$ eran de sexo masculino y $59 \%$ de sexo femenino; el promedio de edad de los participantes fue de 37 años, mientras que la escolaridad media fue de primer año de estudios universitarios.

\section{Instrumento}

Se utilizó un instrumento que mide la $\mathrm{CN}$ y la CS. El instrumento se componía de las siguientes escalas:

Conductas altruistas: consiste en el autoreporte de diez comportamientos dirigidos a asistir o ayudar a los demás, por ejemplo, visitar a personas enfermas en los hospitales, ayudar a los pobres económicamente, apoyar a la Cruz Roja, etcétera. Tapia, Fraijo, Corral, Gutiérrez y Tirado (2006) reportaron el uso de esta escala, obtuvieron indicios de validez convergente y concurrente, así como de confiabilidad con un alfa de Cronbach reportado de .76. Para responder la escala utilizan cuatros opciones: del 0 al 3, donde cero es nunca y 3 es siempre. Algunos enunciados representativos de dicha escala son "Regalar ropa usada que ya no utiliza pero que está en buen estado" y Regalar una moneda a indigentes (pobres en la calle)".

Comportamiento pro-ecológico: contiene quince ítems del instrumento de Comportamiento Pro Ecológico General de Kaiser (1998). Este instrumento incluye el reporte de acciones como reusar, reciclar, conservar la energía, etc., las cuales se responden con una escala del 0 al 3 , donde el cero significa nunca y el 3 siempre. Algunos ejemplos de los ítems pertenecientes a esta escala son "Guardo y reciclo el papel usado" y "Ahorro gasolina, caminando o viajando en bicicleta". Cabe mencionar que Kaiser y Wilson (2000) reportaron un coeficiente de confiabilidad de .72 al aplicar este instrumento. Del mismo modo, Corral, Tapia, Frías, Fraijo y González (2009) obtuvieron niveles de confiabilidad adecuados (.82) al aplicar esta adaptación al español con quince reactivos.

La frugalidad o austeridad se midió con diez ítems que enuncian acciones como comprar lo estrictamente necesario, reusar ropa, comer en casa, etcétera., los cuales se responden con base en una escala tipo likert, cuyas opciones de respuestas van del 0 al 4 , donde cero es totalmente en desacuerdo y 4 totalmente de acuerdo. Este instrumento fue diseñado por Corral y Pinheiro (2004), mostrando también indicadores de validez y un alfa de Cronbach de .60, lo cual indica confiabilidad. Algunos ejemplos de enunciados de esta escala son " $\mathrm{Si}$ mi carro funciona bien, no compro uno más nuevo, aun teniendo el dinero" y "Vivo sin lujos, aunque pueda dármelos".

Para la medición de la equidad se utilizó una escala desarrollada por Tapia, Corral, Fraijo y Tirado (2006), la cual incluye siete ítems, en los cuales se cuestiona a los participantes acerca de comportamientos como proporcionar las mismas oportunidades de educación para niñas $\mathrm{y}$ niños, tratar a ricos y pobres como iguales, etcétera. La escala se responde del 0 al 4, donde 
el cero significa totalmente en desacuerdo y el 4, totalmente de acuerdo. El análisis del instrumento, en opinión de sus creadores, proporcionó evidencia de que presenta validez convergente y un alfa de Cronbach de .75. Los enunciados "Mi pareja tiene el mismo derecho que yo a decidir sobre los gastos en la familia" y "Mi trato para las personas pobres es igual que el que tengo con los más ricos", son ejemplos de los ítems de esta escala.

Por otro lado, la $\mathrm{CN}$ fue evaluada por medio de una escala de catorce ítems que miden el grado en que las personas se sienten, en general, parte del mundo natural. Los participantes respondieron con base en una escala tipo likert, que va del 1 (muy en desacuerdo) al 5 (muy de acuerdo). Algunos ejemplos de los ítems de esta escala son "A menudo me siento en unión con el mundo natural que me rodea" y "Siento como si perteneciera a la Tierra de la misma forma que ella me pertenece a mí". Esta escala fue creada por Mayer y Frantz (2004) y mostró niveles óptimos de confiabilidad (.84) y validez. Para fines de este estudio, se utilizó una adaptación al español de la escala antes mencionada, realizada por Olivos, Aragonés y Amérigo (2011), la cual obtuvo también niveles de confiabilidad (alfa de Cronbach $=.78)$ y validez aceptables.

Finalmente, se recabó la información referente a las variables demográficas como género, edad, escolaridad e ingreso familiar mensual.

\section{Procedimiento}

La aplicación se llevó a cabo con una muestra de la población general de Hermosillo, Sonora. En primera instancia, se pidió el consentimiento de los participantes para responder los instrumentos, más tarde, les fue entregado el instrumento para que fuese respondido a manera de auto-reporte por las personas que accedieron ser partícipes en la presente investigación. La duración promedio de la aplicación fue de 30 minutos y fue realizada en la casa de los participantes, en distintas colonias representativas de la ciudad de Hermosillo, México.

Posteriormente, se conformó una base de datos en el paquete estadístico SPSS, versión 21, donde fueron registrados los datos correspondientes a cada uno de los instrumentos aplicados. Por último, se realizó un análisis de los datos obtenidos en los programas SPSS 21 y EQS 6.1.

\section{Análisis de los datos}

Se obtuvieron las estadísticas univariadas para cada uno de los reactivos de las escalas empleadas. Del mismo modo, se estimó la confiabilidad de cada una de ellas.

Se especificó además un modelo de ecuaciones estructurales para probar la hipótesis de la relación entre la conectividad con naturaleza y la práctica de conductas sustentables, considerando, por un lado, los factores correspondientes a la conducta sustentable (altruismo, austeridad, conducta pro-ecológica y equidad) y, por 
otro lado, la conectividad con la naturaleza. Se obtuvieron también los índices de la bondad de ajuste, tanto estadísticos, como prácticos para verificar la pertinencia del modelo.

\section{Resultados}

La tabla 1 muestra los estadísticos univariados y la confiabilidad obtenida con los datos de los participantes; los resultados reflejan un nivel aceptable en las cinco escalas, con alfas de Cronbach que oscilan entre .60 y .83 . En cuanto a las estadísticas univariadas, la escala con mayor aceptación por parte de los participantes fue la de equidad, con una media de 3.39 (DE $=.52$ ), mientras que la frecuencia de conductas tanto altruistas como pro-ecológicas obtuvieron los valores más bajos del estudio.

La tabla 2 exhibe las correlaciones que se presentaron entre las variables de interés; se destaca que la mayoría tuvo asociaciones estadísticamente significativas, las únicas que no presentan esta relación fueron el altruismo y la austeridad, aunque ambas pertenecen a la

Tabla 1

Estadisticas univariadas y consistencia interna de las escalas de CN y los 4 componentes de la CS

\begin{tabular}{lccccc}
\hline ESCALA/Ítems & Media & D.E & Mín & Máx & Alfa \\
& & & & & \\
\hline Altruismo & 1.77 & 0.48 & 0 & 3 & .73 \\
Conducta pro-ecológica & 1.52 & 0.43 & 0 & 3 & .74 \\
Austeridad & 2.66 & 0.63 & 0 & 4 & .63 \\
Equidad & 3.39 & 0.52 & 0 & 4 & .60 \\
Conectividad & 2.44 & 0.55 & 0 & 4 & .80
\end{tabular}

Tabla 2

Matriz de correlación entre las variables de CN y CS

\begin{tabular}{lccccc}
\hline & CPE & Altruismo & Equidad & Austeridad & Conectividad \\
CPE & 1 & & & & \\
Altruismo & $.47^{* *}$ & 1 & & & \\
Equidad & $.22^{* *}$ & $.27^{* *}$ & 1 & & \\
Austeridad & $.21^{* *}$ & .05 & $.26^{* *}$ & 1 & 1 \\
Conectividad & $.38^{* *}$ & $.35^{* *}$ & $.20^{* *}$ & $.23^{* *}$ & 1 \\
\hline
\end{tabular}

$* \mathrm{p}<.05, * * \mathrm{p}<.01$ 
CS hacen referencia a diferentes componentes, la primera representa al cuidado del ambiente social y la segunda, al ambiente natural. Lo más importante por destacar es que la $\mathrm{CN}$ obtuvo correlación alta y significativa con las cuatro variables que forman la $\mathrm{CS}$.
La figura 1 muestra los resultados del modelo estructural probado. Se revelan cargas factoriales altas y significativas $(p<.05)$ para cada una de las parcelas y su correspondiente factor de primer orden: la conducta pro-

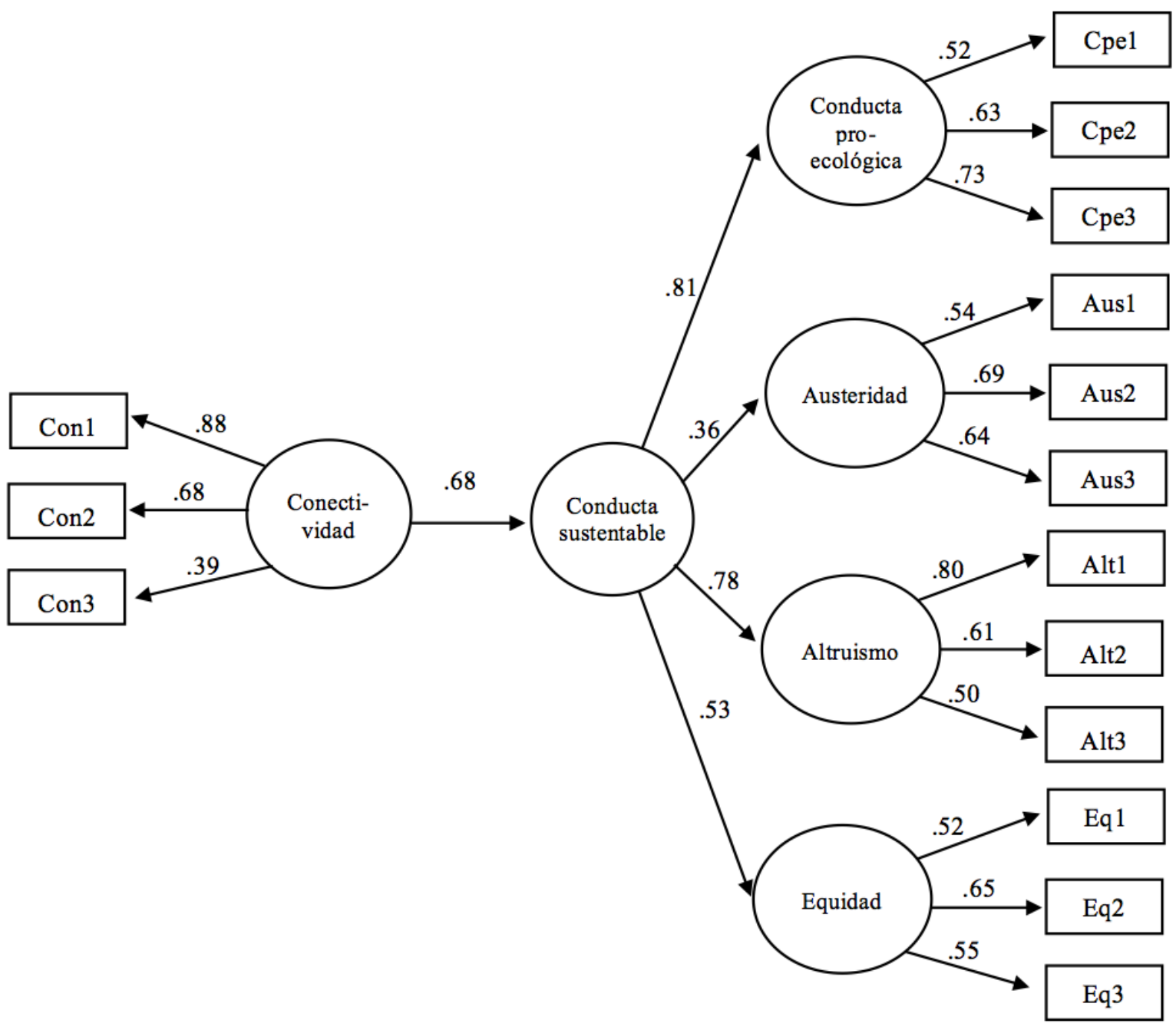

Figura 1. Modelo CN y su relación con la CS. Todos los pesos factoriales y coeficiente estructural fueron significativos $(p<.05)$. Bondad de ajuste: Chi-cuadrada=1036.43 (105 gl), $p<.001$; $B B N N F I=.91, C F I=.93 ; R M S E A=.055$. $\mathrm{CS} R^{2}=.47$. 
ecológica, altruismo, austeridad y equidad, al hablar de la CS y las correspondientes con la conectividad. Del mismo modo, en el caso de las cargas de cada factor de primer orden y su correspondiente constructo de orden superior, denominado CS, también se obtuvieron cargas altas y significativas. Por otro lado, el coeficiente estructural que liga la conectividad con la CS fue alto $(.68)$ y significativo $(p<.05)$. El valor de la $R^{2}$ del modelo fue .47 , lo cual indica que la conectividad explica $47 \%$ de varianza de la CS. Finalmente, los índices de bondad de ajuste señalan que los datos obtenidos sustentan la hipótesis de la relación entre estos constructos.

\section{Discusión}

Como se argumentó en la sección introductoria de este escrito, los problemas ambientales actuales están íntimamente ligados con las relaciones que entablamos con la naturaleza. Según Olivos, Aragonés y Amérigo (2011), las soluciones para la degradación ambiental se encuentran en el estudio de la $\mathrm{CN}$ y el sentimiento de unidad e integración con el entorno natural. Por ello, el propósito de esta investigación fue demostrar que las personas con una mayor $\mathrm{CN}$ naturaleza realizan más $\mathrm{CS}$. Algunos antecedentes en la literatura indicaban que las personas que experimentan este sentido de conectividad se comportan a favor del medio ambiente físico, (Shultz, 2002; Olivos, Aragonés, \& Navarro, 2013; Hinds \& Sparks, 2008), pero faltaba demostrar que la $\mathrm{CN}$ es también capaz de predecir conductas pro-sociales como las altruistas y las equitativas. Como se esperaba, el modelo respaldó la hipótesis establecida para el presente estudio, en el sentido de que a mayor $\mathrm{CN}$ es también mayor la práctica de CS incluyendo las pro-sociales.

Estudios recientes se han dado a la tarea de investigar y caracterizar a las personas inclinadas a seguir estilos de vida sustentables, en términos de rasgos y orientaciones psicológicas (Corral, Tapia, Fraijo, Mireles, \& Márquez, 2008). Estas conductas, por supuesto, incluyen acciones que llevan a la conservación del ambiente físico, así como comportamientos encaminados a proteger a otros individuos y grupos sociales, especialmente los más vulnerables (Corral, 2012). Dichos rasgos, según Corral (2010), incorporan el altruismo, la austeridad, la equidad y la conducta pro-ecológica, mismos que repercuten en el cuidado del medio ambiente físico y social. Los resultados de la presente investigación hicieron énfasis en la inclusión de las cuatro dimensiones o componentes de la CS. Se tomó en cuenta el componente de ayuda a otras personas y no únicamente el de conductas de conservación del medio ambiente bio-físico, mismo que -como se pudo constatar- también es afectado por la $\mathrm{CN}$.

El efecto de la CS se da en diferentes dimensiones, y como argumentan Corral y Pinheiro (2004), esa conducta es necesaria para garantizar el bienestar presente y futuro 
de la humanidad. En este aspecto, es relevante destacar que la frecuencia de conductas reportadas por la población es muy similar para los dos factores principales del comportamiento sostenible: el cuidado del ambiente natural y social; sin embargo, se ubica en un nivel medio, es decir, no se practica con mucha o poca regularidad. En cuanto a la aceptación que tienen las personas en cuestiones del cuidado de la naturaleza y la sociedad, se presenta mayor interés en los demás, puesto que la media de equidad es considerablemente mayor a la obtenida por la escala de austeridad. Los valores que reflejan los estadísticos univariados sugieren que las personas tienen buena aceptación de las dimensiones de la CS (austeridad y equidad), empero, falta que esto se refleje en mayores acciones a favor de la naturaleza y sociedad (altruismo y conducta pro-ecológica). Por otro lado, en los resultados obtenidos con la escala de conectividad, se puede observar que las personas en general están de acuerdo en que los seres humanos somos parte de la naturaleza.

Tanto las correlaciones como el modelo de ecuaciones estructurales, prueban de manera empírica que existe una relación muy estrecha entre la conectividad y las CS, de hecho casi la mitad (47\%) del comportamiento a favor de la sociedad y el medio ambiente es explicado por el nivel de $\mathrm{CN}$ que presentan los individuos. Estos hallazgos comprueban los resultados de estudios previos como el de Olivos, Aragonés y Navarro (2013), con la relación que tiene la conectividad y las conductas pro-ecológicas, aunque es interesante que esta conexión también se traduce en conductas a favor de la sociedad.

Una vez comprobado que las personas con una mayor $\mathrm{CN}$, realizan también más conductas de ayuda al ambiente y a otros seres humanos, sería importante investigar en estudios subsecuentes los efectos psicológicos positivos de la CN. Asimismo, sería deseable instrumentar un mayor número de programas de educación ambiental, en el medio ambiente, donde se haga énfasis en desarrollar en la $\mathrm{CN}$, teniendo en cuenta los resultados alentadores que se presentan en esta investigación.

Del mismo modo, se pudieran realizar estudios que midieran los efectos de la conectividad con personas que viven en el medio rural, y se comparasen con la de aquellas que residen en ambientes urbanos, para medir este nivel de conectividad y sus repercusiones en la práctica de conductas sustentables.

La respuesta a muchas de las problemáticas ambientales y sociales, que tanto se ha buscado en años recientes, pudiera encontrarse en la falta de conectividad y sentido de unidad con el entorno. Es preciso, entonces, trabajar en distintas iniciativas y estrategias, tanto de investigación como de intervención, que amplíen los resultados reportados en este estudio y permitan reconectar a las personas con este sentido de pertenencia a la naturaleza. De esta manera se propiciaría el desarrollo de comunidades con estilos de vida más saludables. 


\section{Referencias}

Brugger, A., Kaiser, F. G., \& Roczen, N. (2011). One for all?: Connectedness to nature, inclusion of nature, environmental identity, and implicit association with nature. European Psychologist, 16(4), 324-333.

Clayton, P. H. (1998). Connection on the ice: Environmental ethics in theory and practice. Philadelphia: Temple University Press.

Clayton, S. (2003). Environmental identity: A conceptual and an operational definition. En S. Clayton \& S. Opotow (Eds.), Identity and the natural environment (pp. 45-65). Cambridge, MA: MIT Press.

Corral, V. (2010). Psicología de la sustentabilidad: un análisis de lo que nos hace pro ecológicos y pro sociales. México, D. F: Trillas.

Corral, V. (2012). Sustentabilidad y psicología positiva: Una visión optimista de las conductas proambientales y prosociales. México: Manual Moderno.

Corral, V., \& Pinheiro, V.(2004).Aproximaciones al estudio de la conducta sustentable. Medio Ambiente y Comportamiento Humano, 5, $1-26$.

Corral, V., Tapia, C., Fraijo, B., Mireles, J., \& Márquez, P. (2008). Orientación a la sustentabilidad como determinante de los estilos de vida sustentables: un estudio con una muestra mexicana. Revista Mexicana de Psicología, 25, 313-327.

Corral, V., Tapia, C., Frías, M., Fraijo, B., \& González, D. (2009). Orientación a la sostenibilidad como base para el comportamiento pro-social y pro-ecológico. Medioambiente y Comportamiento Humano, 10(3), 195-215.

Cronon, W. (1995). Uncommon ground: Toward reinventing nature. New York: W.W. Norton \& Co.

Davis, J. L., Green, J. D., \& Reed, A. (2009). Interdependence with the environment: Commitment, interconnectedness, and environmental behavior. Journal of Environmental Psychology, 29(2), 173-180.

De Young, R. (1991). Some psychological aspects of living lightly: Desired lifestyle patterns and conservation behavior. Journal of Environmental Systems, 20(3), 215-227.

Dutcher, D., Finley, J., Luloff, A. E., \& Johnson, J. (2007). Connectivity With Nature as a Measure of Environmental Values. Environment and Behavior, 39(4), 474-493.

Fisher, A. (2002). Radical ecopsychology: Psychology in the service of life. Albany, NY: State University of New York Press.

Frantz, C., Mayer, F. S., Norton, C., \& Rock, M. (2005). There is no "I" in nature: The influence of self-awareness on connectedness to nature. Journal of Environmental Psychology, 25(4), 427-436.

García, M. Estévez, I., \& Letamendía, P. (2007). El CUIDA como instrumento para la valoración de la personalidad en la evaluación de adoptantes, cuidadores, tutores y mediadores. Intervención. Psicosocial, 16, 393-407.

Gelter, H. (2000). Friluftsliv: The Scandinavian Philosophy of Outdoor Life. Canadian Journal of Environmental Education, 5, 77-92. 
Hedlund, W. A., DeBoer, J., \& Boersema, J. J. (2014). Exploring inner and outer worlds: A quantitative study of worldviews, environmental attitudes, and sustainable lifestyles. Journal of Environmental Psychology, 37, 40-54.

Hernández, S. R., Fernández, C. C., \& Baptista, L. P. (2010). Metodología de la investigación. México: McGraw-Hill.

Hinds, J., \& Sparks, P. (2008). Engaging with the natural environment: The role of affective connection and identity. Journal of Environmental Psychology, 28, 2, 109-120.

I-Chun, T., William, C. S., \& Chun-Yen, C. (2014). Perceptual Evaluation of Natural Landscapes: The Role of the Individual Connection to Nature. Environment and Behavior, 47(6), 595-617.

Kaiser, F. (1998). A general measure of ecological behavior. Journal of Applied Social Psychology, 28, 395-442.

Kaiser, F., \& Wilson, M. (2000). Assessing people's general ecological behaviour: a cross-cultural measure. Journal of Applied Social Psychology, 30, 952-978.

Kellert, S. R. (1997). Kinship to mastery: Biophilia in human evolution and development. Washington, DC: Island Press.

Leary, M. R., Tipsord, J. M., \& Tate, E. B. (2008). Allo-inclusive identity: Incorporating the social and natural worlds into one's sense of self. En H. Wayment, \& J. Bauer (Eds.), Transcending self-interest: Psychological explorations of the quiet ego (pp. 137-147). Washington, DC: American Psychological Association.
Levi, D., \& Kocher, S. (1999). Virtual Nature: The Future Effects of Information Technology on Our Relationship to Nature. Environment and Behavior, 31, 203-226.

Maller, C., Townsend, M., St. Leger, L., Henderson-Wilson, C., Pryor, A., Prosser, L., \& Moore, M. (2008). Healthy parks, healthy people: The health benefits of contact with naturein a park context. Melbourne, Australia: Deakin University \& Parks Victoria.

Mayer, S. F \& Frantz, C. M. (2004). The connectedness to nature scale a measure of individuals' feeling in community with nature. Journal of Environmental Psychology 24, 505-515.

Melson, G. F. (2001). Why the Wild Things Are: Animals in the Lives of Children. Cambridge, MA: Harvard University Press.

Müller, M. M., Kals, E., \& Pansa, R. (2009). Adolescents' emotional affinity towards nature: A cross-societal study. Journal of Developmental Processes, 4(1), 59-69.

Nhat Hahn, T. (1999). Interbeing: Fourteen guidelines for engaged Buddhism. Berkeley, CA: Parallax Press.

Nisbet, E. K., Zelenski, J. M., \& Murphy, S. A. (2009). The Nature Relatedness Scale: Linking Individuals' Connection with Nature to Environmental Concern and Behavior. Environment and Behavior, 41, 715-740.

Oberkircher, L., Shanafield, M., Ismailova, B., \& Saito, L. (2011). Ecosystem and Social Construction: an Interdisciplinary Case Study of the Shurkul Lake Landscape in Khorezm, Uzbekistan. Ecology \& Society, 16, 1-10. 
Olivos, P., Aragonés, J. I., \& Amérigo, M. (2011). The connectedness with nature scale and its relationship with environmental beliefs and identity. International Journal of Hispanic Psychology, 4, 5-20.

Olivos, P, Aragonés, J, \& Navarro, O. (2013). Educación ambiental: itinerario en la naturaleza y su relación con conectividad, preocupaciones ambientales y conducta. Revista Latinoamericana de Psicología, 45, 503-513.

Olson, J., \& Zanna, M. (1993). Attitudes and attitude change. Annual Review of Psychology, 44, 117-154.

Schmuck, P., \& Schultz, P. W.(2002). Psychology of sustainable development. Boston: Kluwer Academic.

Schroeder, H. W. (2002). Experiencing nature in special places. Surveys in the north-central region. Journal of Forestry, 100, 8-14.

Schultz, P. W. (2000). Empathizing with nature: The effects of perspective taking concern for environmental issues. Journal of Social Issues, 56, 391-406.

Schultz, P. W. (2001). Assessing the structure of environmental concern: Concern for self, other people, and the biosphere. Journal of Environmental Psychology, 21, 1-13.

Schultz, P. W. (2002). Inclusion with nature: The psychology of human-nature relations. En P. Schmuck \& P. W. Schultz (Eds.), Psychology of Sustainable Development, (pp 61-78). Norwell, MA: Kluwer Academic.

Schultz, P.W., C. Shriver, J. J. Tabanico, \& A. M. Khazian. (2004). Implicit connections with nature. Journal of Environmental Psychology 24, 31-42.

Tapia, C., Corral, V., Fraijo, B., \& Durón, F. (2013). Assessing sustainable behavior and its correlates: a measure of pro-ecological, frugal, altruistic and equitable actions. Sustainability, 5, 711-723.

Tapia, C., Fraijo, B., Corral, V., Gutiérrez, C., \& Tirado, H. (2006). Validación de una escala de orientación hacia la sustentabilidad. En B. Fraijo, B., S. Echeverría \& C. Tapia (Eds.), Desierto y Mar. Estudios sociales en Sonora, Cd Obregón. México: Instituto Tecnológico de Sonora.

Taylor, C. (1989). Sources of the self: The making of the modern identity. Cambridge: Harvard University Press.

Wells, N., \& Lekies, K. (2006). Nature and the life course: Pathways from childhood nature experiences to adult environmentalism. Children, Youth and Environments, 16, 1-24.

Wilson, E. O. (1983). Biophilia and the conservation ethic. En S. R. Kellert \& E. O. Wilson (Eds), The biophilia hipótesis. Washington: Island Pres.

Recibido el 13 de septiembre de 2016 Revisado el 13 de octubre de 2016 Aceptado el 17 de noviembre de 2016 